\title{
Utilizing Technology to Support and Extend Access to Students and Job Seekers during the Pandemic
}

Daniel Berra

The ongoing pandemic necessitated a reimaging of public library services and resources. Out of this challenge rose opportunities to better serve the needs of our communities during the pandemic and beyond. When our library first closed our doors to the public last March, we began discussions on how the needs of our community have changed. We identified two key groups for whom the pandemic had forced an uncomfortable shift: students suddenly thrust into virtual learning and adults who had lost their jobs. While we continue to serve all members of our community in a variety of ways, we looked to increase support for these specific groups utilizing available technology.

Like many public libraries, the Pflugerville Public Library quickly shifted our service model to include virtual programs, curbside pickup, library cards issued remotely and a focus on electronic resources. Our community is rapidly growing and diverse. Many of our nearly 70,000 residents are frequent users of library services, attend our wide array of programs, hold meetings, study or work inside the building and enjoy both the physical and virtual library collection. The pandemic shift required our talented staff to find ways to provide a similar level of service to a community who heavily utilizes the library.

For both students and job seekers, we took steps to alleviate some of the difficulties the building's closure caused by utilizing existing technology. We worked with the city's IT department to extend the library's Wi-Fi to cover the entire parking lot, allowing for 24-hour access. We also utilized our existing print from your own device system to allow library users to submit print jobs and then pick them up through our curbside service. We added additional Wi-Fi hotspots available for checkout to ensure access at home for those lacking internet. Since these services were already offered to some degree, the expansion of access was relatively easy to implement.

For students we drew upon our existing relationship with the Pflugerville Independent School District (PFISD) to provide support and extend access. We expanded the offering of our special DigiT cards, which allow students to sign up for an account giving them access to all of our electronic resources and Wi-Fi hotspots. The school district's librarians handle the signups and then submit the forms so we can set up the accounts then contact students by email or phone. We further extended access to eBooks by working with the district and our vendor Overdrive, to provide a direct way for students to browse and check out through the district's own eBook App. This allows students to seamlessly see both of our collections, significantly increasing their reading options and removing barriers to access.

On the support front, we utilized a portion of the city's CARES Act funds directed toward the library to launch a live, virtual tutoring service called Brainfuse HelpNow. Students of all ages have anonymous access to tutors from home seven days a week, as well as additional homework

Daniel Berra (danielb@pfulgervilletx.gov) is Assistant Director, Pflugerville (Texas) Public Library. (C) 2021.

"Public Libraries Leading the Way" is a regular column spotlighting technology in public libraries. 
support resources. This piece meshes nicely with some of our virtual programming for teens, like our SAT and ACT practice tests and other test- and career-preparation e-resources.

Recognizing the pandemic's impact on the economy, and how this directly affects our community, we worked to prioritize support for the unemployed and under-employed. We added a resume review/job-search coaching service led by two of our circulation staff members. We utilized another portion of our CARES Act funds to offer Career Online High School, providing adults with access to an online program to obtain their high school diploma. We also began lending laptops for home use to ensure access to necessary technology.

Some of our support was already in place before the pandemic began, and we made a significant marketing push to highlight these e-resources. For instance, we partner with the Pflugerville Community Development Corporation to provide the online training resource Lynda.com (soon to be LinkedIn Learning). We saw a large increase in usage particularly in the first few months of the pandemic as community members looked to add employable skills to their toolboxes. We also created a page on our website with all of our job search assistance resources and services highlighted in one place.

While the main emphasis of these efforts utilizes technology, serving the needs of the entire community also requires supporting those who are generally less connected. We have to balance our digital expectations with something more tangible, recognizing many library users still utilize the library in a more traditional way. For students, our Senior Youth Services Librarian partnered with PFISD for a book give away in conjunction with the district's food distribution program to get books in the hands of children for the summer. We also began distributing "Care Kits" through our curbside service that include personal grooming products and cold weather gear for anyone in need.

While 2020 featured the addition of many new services or significant expansion of existing ones, we are focused in 2021 on increasing our marketing efforts for these offerings. Relying too heavily on digital forms of communication can limit the impact of our services. For instance, if we want to let people who do not have access to the internet at home know we have Wi-Fi hotspots and laptops available for checkout, then spreading the word through our standard methods of social media, website, and email will prove ineffective. With the building currently closed to the public, we face an additional barrier to communication. To help alleviate some of this, we have created a Job Search Assistance flyer that we are distributing at places like local food pantries. We plan to expand on similar methods of marketing throughout the year.

While positive feedback is often hidden from libraries since we prioritize patron privacy and anonymity, we have received a few specific stories that highlight our impact. Our first scholarship recipient for Career Online High School shared how the opportunity to obtain her high school diploma will open up new professional avenues and erase the stigma of having not completed high school. Another community member who took advantage of our job search coaching to prepare for an interview expressed gratitude to the library staff who helped increase his employment chances. We also see resumes and homework assignments printed through our virtual printing service, hear from parents with children utilizing hotspots for virtual schooling, see cars in the parking lot using the extended Wi-Fi and track statistics showing a large increase in the usage of our electronic resources. 
The ongoing pandemic necessitated a re-imagining of library services. The needs of our community changed and we set out to find ways to provide assistance to those who need it the most utilizing technology, while remaining mindful of those who are not as comfortable in the digital age. The combination of utilizing technology to address the current needs and expanding access to this technology, has allowed us to better serve the community. We are in the process now of evaluating all of our changes to determine which ones will continue even after the pandemic ends. We already know that we will keep our methods of extending access like the expanded Wi-Fi availability, laptops for checkout, DigiT cards for students and the seamless connection to our eBook collection for PFISD. In the area of support, we will continue to offer Career Online High School, Brainfuse HelpNow for virtual tutoring, and our resume review/job search coaching service.

Public libraries are well positioned to innovate and adjust to changes in society. It is one of the things we do extremely well, out of necessity, but also out of a deep desire to serve our communities. All of the shifts the Pflugerville Public Library made related to supporting students and job seekers drew upon existing technology and available resources. What changed was the areas on which we chose to focus our efforts. By prioritizing support and access while pinpointing the needs of the moment, we found ways to better serve our community within the context of everything else we provide. While the jury is still out on how successful some of these initiatives will prove, we already know that many of these changes will continue long after the pandemic ends. 